\title{
EDUCACIÓN HACKER: UNA EXPRESIÓN EMERGENTE DE LA PEDAGOGÍA CRÍTICA PARA LA SOCIEDAD EN RED
}

Alexandre Garcia Aguado Isabel Alvarez Canovas ${ }^{(*)}$

\section{INTRODUCCIÓN: LA EDUCACIÓN EN TIEMPOS DE PROTOCOLOS, ALGORITMOS Y REDES SOCIALES}

Cuando hablamos de la relación entre las sociedades y las Tecnologías de la Información y Comunicación (TIC), nos llaman la atención dos preocupaciones particulares. La primera es en relación el acceso a estas tecnologías, pues $43,2 \%$ de la población mundial no tiene acceso a Internet (INTERNET WORLD STATS, 2019), siendo que tal exclusión trae consigo el peso de la inaccesibilidad a importantes oportunidades y dinámicas sociales y económicas que cada vez más ocurren por allí. La segunda preocupación, la cual vamos a ver más específicamente en las siguientes líneas, está relacionada con la calidad de las interacciones que ocurren en la Internet, representadas por las diferentes formas de apropiación, los acuerdos establecidos entre los actores que pueblan el ciberespacio y especialmente por nuestra interacción con los algoritmos y protocolos que manejan nuestros datos personales.

En 1989 cuando Tim Berners-Lee presentó su propuesta original de lo que seria la worldwide $w e b^{1}$, su deseo era que esa gran red pudiera ser una plataforma abierta, para conectar a las personas esparcidas por el mundo para que juntas pudiesen buscar soluciones a los grandes problemas que fragilizan a la humanidad, sin embargo, últimamente, la forma con que las grandes corporaciones y los estados se han apropiado de la Internet ha sido motivo de preocupación para elle. Según Berners-Lee, nosotros hemos perdido el control de nuestros datos personales y como consecuencia, surgió una sofisticada industria que a través de algoritmos que se basan en conjuntos de datos personales, conducen campañas políticas con anuncios individuales dirigidos directamente a cada persona (BERNERS-LEE, 2017).

\footnotetext{
${ }^{(*)}$ Alexandre G. Aguado. Instituto Federal de Educação Ciência e Tecnologia de São Paulo. Orcid: <https://orcid.org/ 0000-0002-1874-4679>.

Isabel A. Canovas. Profesora asociada en el Departamento de Pedagogia Sistemàtica i Social de la Facultad de Ciências de la Educación - Universitat Autònoma de Barcelona. Orcid: 〈https://orcid.org/0000-0001-9488-9960>.

${ }^{1}$ Es importante aclarar que Internet y web no son lo mismo. Internet es la red de redes que conecta miles de redes locales en todo el mundo, mientras que la web, que se usa de la Internet, és de acuerdo con su definición original, "una iniciativa de recuperación de información hipermedia con el objetivo de dar acceso universal a un gran universo de documentos". Para más detalles, visite: $<$ https://www.w3.org/MarkUp/html-test/misc/guide.html>
} 
Un claro ejemplo de esto son los recientes casos revelados por una serie de reportajes del periódico británico The Guardians ${ }^{2}$ y que actualmente reverberan a través del documental The Great Hack, publicado en 2019 por Netflix, donde se revelan los mecanismos avanzados utilizados por la consultora política Cambridge Analytica para determinar la personalidad de las personas y así ejecutar estrategias de manipulación para favorecer la elección de sus clientes.

Como revela el ex empleado de la consultora, Christopher Wylie para la periodista Carole Cadwalladr, a través de la recogida de dados de los perfiles de Facebook de millones de personas fue posible utilizar las informaciones privadas y personales para crear avanzados perfiles psicológicos y políticos y luego bombardearlos con anuncios diseñados específicamente para su perfil específico, logrando así una gran precisión en el objetivo de llevar el voto al lado deseado. Así fue en la campaña de Ted Cruz, Donald Trump y Brexit. Detrás de este movimiento está la figura de Steve Bannon, vicepresidente de Cambridge Analytica y, en particular, el articulador de un movimiento mundial de extrema derecha (CADWALLADR, 2018).

También tomando como materia prima datos personales y aparentemente triviales de las personas, otra dinámica que preocupa a Tim Berners-Lee es la explotación comercial en la red, donde los algoritmos de inteligencia artificial, cuidadosamente construidos para distraernos, nos llevan a sitios con información confusa o falsa, donde aquellos con intenciones maliciosas pueden obtener alguna ganancia financiera (BERNERS-LEE, 2017). Como presenta Zuboff (2015, p. 76), sufrimos las consecuencias del capitalismo de vigilancia, o sea, una "nueva forma de capitalismo de información que tiene como objetivo producir lucro y control del mercado", do cual no es una opción simple tentar no hacer parte, pues como señala Evangelista (2018), existe una presión social para usar estos sistemas, incluso, en el mundo del trabajo donde una serie de interacciones ocurren exclusivamente en determinadas redes sociales.

Todos estos aspectos y preocupaciones apuntan a nosotros la complejidad que hay en nuestro contexto contemporáneo en la relación entre sociedad y las TIC, así como, también nos despierta sobre la importancia de las estrategias que ayudan a las personas a apropiarse de las tecnologías no solo instrumentalmente sino también desde una perspectiva crítica, tomando conciencia de las potencialidades y consecuencias de tal apropiación. En este punto que sostenemos que es en la educación que encontramos un espacio de posibilidad, favorable a estas estrategias tan necesarias en nuestro contexto contemporáneo, siendo un espacio "de empoderamiento de los sujetos para una formación de la ciudadanía, comprometida y activa con el contexto en que viven

\footnotetext{
${ }^{2}$ Disponible en: <https://www.theguardian.com/news/series/cambridge-analytica-files>.
} 
estos sujetos" (LAPA; PRETTO, 2019, p. 35). Así, concordamos que la escuela no eres el único espacio para el desarrollo de una educación emancipadora y democrática, pero si, que eres el espacio adecuado, donde su rol de lugar de conexiones, democratización y inclusión, debe ser rescatado (LAPA; PRETTO, 2019).

En este punto es importante recordar que la escuela es también un espacio de disputa entre diferentes perspectivas de la apropiación tecnológica. Un ejemplo de esto ha sido la gran expansión de los productos Google para educación, que más allá de las diversas posibilidades que permite, tienen como característica de uso, la necesidad de los usuarios crearen una cuenta, teniendo que informar algunos datos personales. Como los algoritmos detrás de estas soluciones tecnológicas no están abiertos, la auditabilidad, que permitiría saber qué se hace con los datos recogidos no existe (SINGER, 2017). Mirar este tipo de apropiación tecnológica de manera crítica es imprescindible para una educación que busca ser emancipadora. Creemos que a medida que aceptamos dócilmente que ciertas empresas pueden debilitar nuestra privacidad y de nuestros estudiantes, comunicamos a la sociedad que esto es normal y no necesita cambiar.

En este punto, la concepción que tenemos de educación se acerca da revelada por los teóricos y prácticos de la pedagogía crítica, los cuales han buscado comprender la escuela en el ámbito social, cultural, político y económico, siendo un espacio que puede ser visto al mismo tiempo como un mecanismo de clasificación, favoreciendo a determinados grupos con base en la raza, clase o género así como un espacio agenciador de empoderamiento. Comprender, desvelar, criticar y modificar las estructuras de poder que de alguna forma oprimen determinados grupos es parte significativa de lo que necesitamos hacer (MCLAREN, 2005).

A pesar de considerar la tecnología como un aspecto entrelazado con el desarrollo humano, el movimiento de la pedagogía crítica desde el siglo XX, representado por Paulo Freire, Henry Giroux, Peter Mclaren y otros, se ha centrado principalmente en otros temas como la alfabetización, la democracia y la conciencia crítica. La mirada a la realidad tecnosocial ha sido cada vez más necesaria en la pedagogía crítica contemporánea, una mirada que no es utópica, pues no cree que las tecnologías con el tiempo resolverán todos los problemas humanos y también no es distópica, creyendo que llevará la humanidad al armagedón. De hecho, la superación de una visión determinista de la tecnología ha ocurrido en las corrientes principales de la pedagogía crítica en parte gracias a las obras de los críticos freirianos (MCLAREN; ESCAÑO; JANDRIĆ, 2018).

Una educación que permita al hombre la discusión valiente de su problemática. De su inserción en esta problemática. Que le advirtiese de los peligros de su tiempo, para que, consciente de ellos, ganara la fuerza y el coraje de luchar, en vez de ser llevado y 
arrastrado a la perdición de su propio yo, sometido a las prescripciones ajenas. Educación que lo pusiera en diálogo constante con el otro. Que lo predispase a constantes revisiones. Al análisis crítico de sus hallazgos. (FREIRE, 1967, p. 90).

Comprender estas dinámicas a partir de una visión armónica que sea verdaderamente humanista y no niegue que somos una sociedad altamente tecnológica, que supere el "falso dilema humanismo-tecnología" (FREIRE, 1967, p. 97), es esencial para una educación que busca ser verdaderamente emancipadora en tiempos de Internet, redes sociales, algoritmos y protocolos. En esta línea, concordamos con Mclaren, Escaño y Jandrić (2018, p. 39) que en la sociedad en red, la pedagogía crítica se acerca y se funde con las culturas digitales, donde "esta poderosa mezcla acepta la diversidad y la complejidad de la vida humana en entornos digitales, y mantiene firmemente la tradición de lucha por la acción humana, la emancipación y la libertad”.

Así, la invitación que hacemos es que volvemos nuestra mirada hacia el modo de hacer educación de un movimiento contracultural, con fuerte enrraigamiento en las culturas digitales, pautado en valores y objetivos que se confunden con aquellos presentados por los prácticos y teóricos de la pedagogía crítica: el movimiento hacker (ESCAÑO, 2018a).

\section{EL MOVIMIENTO HACKER}

Era el final de los años 50 en el Massachusetts Institute of Technology - MIT donde un grupo de jóvenes curiosos y aficcionados formaban el Tech Model Railroad Club - TMRC, "Peter Samson y sus amigos habían crecido con una relación específica con el mundo, donde las cosas sólo tenían significado si descubrían cómo funcionaban" (LEVY, 1994, p. 13). Es a partir de la inquietud de Peter Samson y sus amigos en relación a las antiguas máquinas que funcionaban como ordenadores en el edificio 26 del MIT que Steven Levy (1994) comienza a narrar la historia de esa primera generación de hackers.

También asociada a las elaboradas bromas que los estudiantes del MIT hacían, la palabra hack, era constantemente utilizada por los miembros del TMRC para hacer referencia a los productos y proyectos que en su construcción involucra acciones placenteras para sus ejecutores, o sea, que no eran acciones ejecutadas simplemente para cumplir una tarea que alguien mandó. Así, aquellos que conseguían superar los desafíos, rompiendo las barreras y los límites, eran llamados por el grupo de hackers (LEVY, 1994, p. 13).

El término hacker fue posteriormente eternizado en el jargon file, un diccionario de términos relacionados con la cultura hacker, construido por Eric Raymond. Según Raymond (2004) 
un hacker puede ser un experto o entusiasta de cualquier tipo y área. Él también asocia el término a aquellas personas que les gustan superar desafíos intelectuales y superar las limitaciones con creatividad.

El jargon file ha tenido un papel importante también en el proceso de descriminalización del término hacker. Fue a través de él que Raymond (1999) buscó aclarar la visión de que aquellos que cometen crímenes virtuales en realidad no son hackers sino crackers $^{3}$. Lo que diferencia al hacker del cracker es la ética presente en las comunidades hacker: la ética hacker. Desde el punto de vista de las capacidades técnicas, ambos probablemente poseen semejanzas, pero los hackers siguen un código de ética orientado a la construcción y no la destrucción (HIMANEN, 2001).

En la práctica esta dicotomización no es tan simple. Por ejemplo, un grupo que revela un conjunto de tramas inmorales entre determinados políticos, sin embargo, para hacerlo, tuvieron que promover un determinado ataque a los móviles de esos actores públicos, son crackers, porque hirieron la privacidad de esos políticos o son hackers, porque contribuyeron fuertemente con su sociedad al traer a luz esas actividades excusas? Este es un interesante dilema.

Fue Levy (1994, p. 32-41) uno de los primeros investigadores sobre la cultura hacker a sistematizar un conjunto de instrucciones que revelan aspectos relevantes sobre la forma de actuar de los hackers. Son las siguientes:

1. El acceso a las computadoras y cualquier cosa que pueda enseñarle algo sobre cómo el mundo funciona debe ser ilimitado y total. Siempre a través de un enfoque "hands-on";

2. Toda información debe ser libre para que se pueda mejorar las cosas desde un intercambio libre de informaciones;

3. Desconfíe de las autoridades y promueva la descentralización;

4. Los hackers deben ser juzgados por sus hackings y no por su grado de instrucción, edad, raza o posición;

5. Es posible crear arte y belleza con un ordenador;

6. Los ordenadores pueden cambiar su vida para mejor y

7. Al igual que la lámpara de Aladino, usted puede usar esto (la ética hacker) para hacer lo que quieras.

Estos aspectos son citados por Levy (1994) teniendo como contexto la primera generación de hackers que se reunieron alrededor de la computadora TX-0 al final de los años 50 y principios de los 60. Se puede ver a partir de ahí la presencia de valores como el acceso libre, la aprendizaje

\footnotetext{
${ }^{3}$ Definición disponible en <http://www.catb.org/jargon/html/C/cracker.html〉.
} 
"hands-on", la mirada crítica en relación a las autoridades y la valorización de las personas por aspectos ajenos a raza, color, edad o otros irrelevantes.

El tiempo fue pasando y esa cultura se fue expandiendo a otros contextos y moldeando las relaciones de muchas comunidades, como fue el caso del movimiento del software libre. Hasta principios de la década de 80 era común para los programadores la práctica de compartir códigos entre sí para que uno pudiera mejorar y aprender con lo que el otro hizo, sin embargo, las grandes empresas comenzaron a mercantilizar esas actividades y una de las bases de esa mercantilización era mantener los códigos-fuente ${ }^{4}$ como secretos, protegiéndolos con copyrights. Como respuesta a este movimiento de cierre y explotación comercial de los softwares, surgió el Movimiento del Software Libre, como un movimiento político de base tecnológica, teniendo como su principal interlocutor, Richard Stallman, que con otros hackers inició el desarrollo de un nuevo sistema operativo basado en UNIX y bautizado de GNU (GNU is Not Unix), que en pocas semanas dio origen a GNU/Linux a través de los trabajos de Linus Torvalds y otros hackers (AGUADO, 2012).

Este fue el inicio de un gran movimiento que contribuyó fuertemente para las TIC alcanzar lo que son actualmente. Muchos hackers estaban involucrados en el desarrollo de los protocolos que hacen parte de Internet, así como la worldwide web, fue inspirada en un proyecto de Ted Nelson sobre hipertextos y desarrollada por Tim Berners-Lee junto a otros hackers que forman actualmente el World Wide Web Consorcio (CASTELLS, 2003).

En su libro titulado La ética de los hackers y el espíritu de la era de la información, Pekka Himanen retoma la famosa obra de Max Weber, La ética protestante y el espíritu del capitalismo, para definir las bases de la ética hacker, a cual contrasta con las bases capitalistas que permean nuestra sociedad (HIMANEN, 2001).

\footnotetext{
${ }^{4}$ De acuerdo com el jargon file, código fuente eres "the preferred human-readable and human-modifiable form of the program". Más detalles en: http://catb.org/jargon/html/S/source.html.
} 
Para Himanen (2001), la ética de los hackers puede ser comprendida a partir de la forma en que éstos se relacionan con el trabajo, el dinero y la red (Figura 1).

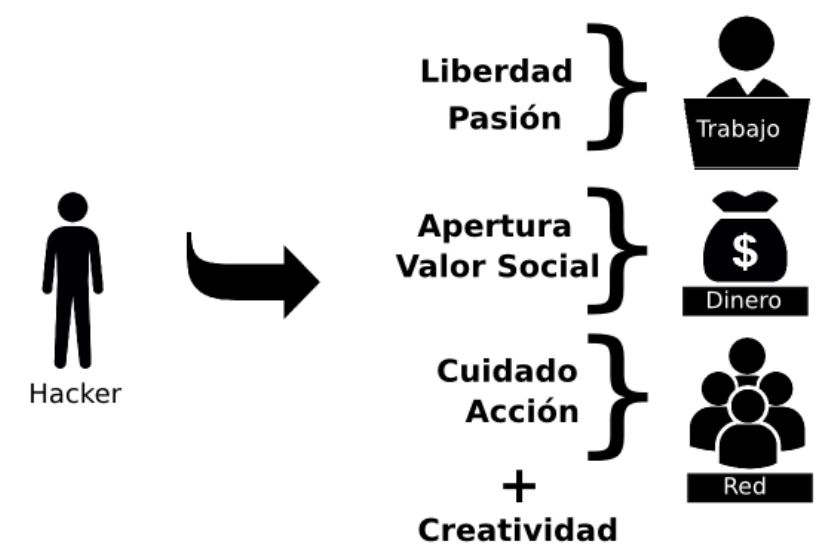

Figura 1. Pilares de la Ética Hacker según Himanen (2001)

El trabajo para los hackers, no debe ser algo penoso, un sacrificio, sino algo que se haga por pasión, o como Linus Torvalds suele decir, por diversión. Para que esto ocurra, la libertad es un aspecto fundamental, sea en el hacer, en el ser o en el vivir. El dinero, íntimamente relacionado con el trabajo, no debe ser el objetivo final de las acciones de los hackers, sino la consecuencia de un trabajo que de alguna forma proporcionó valor social. Es común en el capitalismo de fuerte competición que la información y las creaciones se protegen a siete claves, pues, son un diferencial competitivo que se convierte en lucro. Esta lógica no se aplica a los hackers, para los cuales la apertura es un valor insustituible.

Los hackers, a pesar de su fama de antisociales, se relacionan mucho en red, a través de las comunidades virtuales o presenciales. Esta relación en red es pautada por el cuidado mutuo y por una postura activa. Según Himanen (2001), cabe al hacker la experiencia de crear y recrear el mundo y, así, nos presenta lo que llama de valor supremo de la ética hacker: la creatividad. La creatividad aquí citada no es sólo la expresión de una persona que tiene ideas buenas, pero más que eso, se refiere al uso imaginativo de las habilidades que cada uno tiene para que, superándose a sí mismo, pueda crear de forma a contribuir genuinamente a el mundo, su comunidad y red.

Actualmente, las comunidades de software libre continúan siendo una de las principales vertientes del movimiento hacker. Es muy difícil contabilizar cuántas comunidades existen alrededor del mundo, pero para tener una idea, un único repositorio de comunidades, el sourceforge, posee más de 430.000 proyectos. Son más de 3.7 millones de personas colaborando entre sí en torno a la construcción de software libre. Las comunidades de software libre no están compuestas 
solamente por programadores pero traductores, revisores, administradores y personas de las más diversas áreas (SOURCEFORGE COMMUNITY, 2019).

La emergencia del movimiento hacker también posibilitó la base cultural para la creación de los hackerspaces y hacklabs. Los hacklabs, por haber surgido en un contexto marcado por movimientos anticapitalistas y de lucha por el acceso a las infraestructuras de comunicación emergentes, crecen teniendo como aspectos centrales la adopción de redes de computadoras y tecnologías de medios para usos políticos, posibilitando el acceso a las tecnologías por parte de los menos favorecidos y también defendiendo fuertemente la creatividad popular (MAXIGAS, 2012, p. 5). Los hacklabs, según Maximas (2012, p. 11) son un ejemplo del extremo espectro político en relación a los laboratorios comunitarios y cerca de ellos están los hackerspaces, que son "creados por hackers para hackers con la principal misión de apoyar el hacking”. Actualmente se enumeran ${ }^{5}$ 1416 espacios activos.

Teniendo la esencia de las primeras generaciones de hackers presentadas por Levy (1994) y la ética hacker estudiada por Himanen (2001), otras comunidades también surgieron. Las comunidades de BioHacking han ganado cada vez más participantes, juntando biología con cultura hacker. Los movimientos por la cultura libre, música libre, foodhacking y hasta las comunidades que desarrollan Recursos Educacionales Abiertos (REA) son expresiones de ese movimiento plural y heterogéneo.

\section{LA EDUCACIÓN HACKER: CONTEXTOS, INVESTIGACIONES Y PROYECTOS}

Cuando comprendemos educación como una matriz de esperanza, un proceso de construcción autónoma del inacabado, un proceso de humanización de sí y de su entorno (FREIRE, 1967, 2000), se percibe que existe una gran proximidad de ella con la cultura hacker.

A pesar de todavía poco explorada, esa relación, como campo de investigación ha despertado el interés de muchos investigadores en Brasil y en el mundo. A fin de obtener un retrato actual sobre el tema, he realizado diversas investigaciones en bases de datos científicas, foros y mecanismos de búsqueda en la Internet, teniendo como palabras clave “cultura hacker" y "educación”, juntas. El recorte temporal fue de 6 años, o sea, se consideraron iniciativas prácticas, artículos científicos, relatos personales e investigaciones de maestría y doctorado de 2013 a 2018.

\footnotetext{
${ }^{5}$ Consulta ao sitio web en 13/09/2019 através del link <https://wiki.hackerspaces.org/>. List_of_Hacker_Spaces.
} 
Entre los investigadores que se han dedicado al tema en Brasil, con destaque está el Profesor Nelson de Luca Pretto de la Universidad Federal de Bahía, institución que se ha destacado con grupos de estudios y proyectos en esa línea. En una de sus obras, Pretto (2015, p. 19) elabora los principios para él de una educación hacker en las escuelas. Aspectos como el incentivo a una mirada crítica, descentralización del aprendizaje, comprensión de la diversidad y incentivo del error como parte relevante en el proceso de aprendizaje son centrales en la construcción de Pretto (2015, p. 19).

Una propuesta de formación docente para la educación hacker fue elaborada por Aguado et al (2015). En el trabajo citado, pasión, libertad, valor social, apertura, acción, cuidado y creatividad se consideran ejes referenciales a la formación del profesor hacker, a partir de cuestionamientos que pueden desencadenar un camino de autorreflexión.

La educación hacker ha sido pauta de importantes encuentros entre investigadores. Los Seminarios Nacionales de Inclusión Digital (SENID) son un ejemplo de ellos. La segunda edición de ese seminario, ocurrió en 2013 y tuvo como tema Por una cultura hacker en la educación. Durante tres días de encuentro, entre talleres, charlas y paneles, investigadores reflexionaron en colaboración sobre los caminos posibles para la educación a partir de enfoques que involucren el empoderamiento tecnológico y los presupuestos presentes en la cultura hacker.

Fuera de Brasil algunas iniciativas de investigación también han surgido. El profesor de la Universidad de Sevilla, José Carlos Escaño Gonzalez, es uno de los investigadores que ha trabajado con el tema. En 2013 él dirigió un documental llamado Cultura Libre y Educación Hacker ${ }^{6}$. En el documental, los investigadores comparten sus percepciones sobre educación y la necesidad de hackear la escuela, creando un contexto abierto, creativo, crítico, emancipador, conectado en red, para la construcción de la autonomía.

En el campo de la cultura libre y educación en artes, Escaño (2018a, p. 85) sintetiza algunas características de una educación hacker en su visión, enfatizando características como el compromiso activo en la construcción del mundo, conocimiento compartido, incentivo al pensamiento crítico, acción creativa y la pasión educativa. En otra de sus obras, Escaño (2018b) acerca la educación hacker de la pedagogía crítica, valorando en especial valores como la criticidad, intercreatividad, acceso libre, construcción colaborativa del conocimiento, desarrollo en común, en lo común y en protección de los comunes.

\footnotetext{
${ }^{6}$ Disponible en: <https://vimeo.com/74514091>.
} 
El ecosistema de aprendizaje ha sido observado también por investigadores en los hackerspaces (BURTET, 2014; MENEZES, 2018; SCHROCK, 2014). Mientras Schrock (2014) tiene como contexto de investigación el hackerspace GeekSpace en América del Norte, Burtet (2014) se emergió en el MateHacker en Porto Alegre, Brasil. En los relatos de Burtet (2014) encontramos de forma detallada aspectos de la rutina de un hackerspace. En el ámbito del aprendizaje de los hackers la investigadora enfatiza el compartir de saber en la práctica, la postura crítica, las decisiones democráticas y el orden que se da en medio del caos.

En sus trabajos Schrock (2014, p. 14) observa que en el hackerspace se destacan características como el autodidactismo y el aprendizaje colectivo a partir de proyectos comunes. El investigador resalta la importancia de un ambiente orientado por proyectos para impulsar el crecimiento y la socialización a lo largo del tiempo.

Sistematizar los elementos de la pedagogía presente en los hackerspaces fue el objetivo de Menezes (2018) en su tesis, en la que representó a través de una pirámide una pedagogía de compromiso multifacético, con cuatro caras específicas e interrelacionales: una técnica, una afectiva, una idearia y una política.

Así como estos trabajos citados anteriormente, algunos proyectos de los cuales estamos más cercanos, tienen por objetivo aproximar la educación hacker de las escuelas y ha contribuido con una percepción más empírica en la comprensión de la educación hacker. Uno de esos proyectos, el joven hacker, fue creado "a partir de la confluencia de intereses de entusiastas del software libre, de la cultura libre y del movimiento hacker e investigadores de la universidad". Uno de sus principales objetivos es el de contribuir a la formación de una juventud autónoma y protagonista tecnológicamente en la sociedad (AMIEL et al., 2015, p. 3).

En una primera experiencia en 2014, se hizo el piloto del proyecto en Campinas-SP. Nueve jóvenes de escuelas públicas, con edades entre 16 y 18 años, participaron del piloto. Esta primera experiencia, cuyo objetivo fue insertar a estos jóvenes en el mundo de la programación, generó una serie de contribuciones que permitieron el perfeccionamiento del proyecto para los próximos años.

En 2015, dos ediciones ocurrieron de forma concomitante: una en la ciudad de Campinas y otra en la ciudad de Capivari, como un proyecto de extensión del Instituto Federal de São Paulo. En las dos ediciones, el público principal fueron jóvenes carentes de las ciudades, que a través de dos encuentros semanales, tuvieron la posibilidad de acercarse a las tecnologías de la información y comunicación y experimentar los aspectos de la cultura hacker. El período de encuentros fue organizado en cuatro módulos: 1) introducción al ordenador y la lógica; 2) conceptos básicos de 
programación web; 3) programación con python y 4) proyecto colaborativo social. Las características centrales de la cultura hacker sirvieron de guía para la dinámica de los encuentros. Las cuestiones como: horarios, reglas de convivencia, cambios de itinerario, adecuación del espacio físico e incluso las dinámicas de los encuentros, eran todos decididos colaborativamente, dando así libertad a los participantes y posibilidad de elegir sus rumbos.

La edición de Capivari, en 2015, ocurrió en el Telecentro del barrio São João, uno de los más necesitados de la ciudad. Como el telecentro necesitaba adecuaciones y reformas, se propuso en una de las assembleias que los jóvenes y adolescentes del proyecto pudieran ejecutar las reformas, de forma colaborativa, ejercitando así el protagonismo peculiar de las comunidades hacker. De esta forma, el Telecentro recibió una nueva pintura y un nuevo diseño. El aprendizaje técnico fue realizado de forma que posibilitó contribuciones a la comunidad local. Cada participante montó su propio ordenador, los cuales quedaron después para la comunidad. La red de computadoras del Telecentro fue rehecha por los participantes, permitiendo que actualmente, este espacio sea utilizado para cursos y otras atenciones a las personas del barrio. En el módulo final del proyecto, en grupo, los participantes tenían que desarrollar alguna solución tecnológica que contribuyese a la comunidad. De esta acción, surgieron nuevos juegos e incluso un sitio para acercar a los vecinos del barrio del servicio público local.

El éxito del proyecto hizo que en 2016 la ciudad de Rafard también se interesara por su realización. Dos ediciones ocurrieron este año: una en la ciudad de Rafard y otra en Capivari. Se atendieron aproximadamente a 40 jóvenes y adolescentes, alumnos de las escuelas públicas de la región. En períodos cortos, como un semestre, fue posible percibir que jóvenes y adolescentes que nunca tuvieron una experiencia técnica con la informática, lograron desarrollar sus primeros softwares con una calidad significativa y más relevante que eso, dominando la tecnología en términos sociales, madura noción de que la apropiación de las tecnologías puede contribuir a la sociedad, interfiriendo en las dinámicas de poder presentes en el mundo.

El Proyecto Joven Hacker nos ha dado pistas de que la educación hacker esta íntimamente ligada a la creación de un ecosistema de emancipación, incluso, con potencial para motivar la toma de conciencia de las personas en relación a las dinámicas de poder que los oprimen.

Aún más cerca de la educación formal, se inició en 2017 el proyecto Conexão Escola Mundo $^{7}$. Él nació con el objetivo principal de crear y experimentar una metodología de intervención en la escuela para la formación en derechos humanos dentro de la perspectiva de la

\footnotetext{
${ }^{7}$ Más detalles en: 〈www.escolamundo.ufsc.br〉.
} 
educación hacker. Financiado por el Consejo Nacional de Desarrollo Científico y Tecnológico de Brasil, es desarrollado en cooperación por investigadores de la UFSC y la Universidad Federal de Bahía.

El proyecto funciona como una integración entre universidad y escuela donde investigadores, becarios y maestros actúan juntos tanto en las acciones de levantamiento de demandas en relación a los derechos humanos que necesitan ser trabajadas en cada clase como en la definición de las acciones que serán desarrolladas a lo largo del año. Estos grupos de acción tienen en ellos la representatividad de los estudiantes, lo que posibilita la horizontalidad en la planificación en lugar de una jerárquica rígida.

A partir de esa experiencia tendremos en un futuro próximo, datos más concretos sobre las características de la educación hacker en el contexto escolar, limitaciones para su vivencia y las contribuciones de esa experiencia para la emancipación y empoderamiento de las personas y comunidades.

\section{REFLEXIONES FINALES: SÍNTESIS Y HORIZONTES}

Sería un gran error decir que solo hay una forma de promover la educación hacker. Hay varias comunidades, dispersas por todo el mundo, con sus especificidades y características culturales locales que varían de acuerdo con numerosos factores, pero, de hecho hay principios que, incluso si no integran completamente el ecosistema de todas las comunidades, son parte del ideal de esa cultura, es decir, están en el horizonte de aquellos que buscan incorporar cada vez más la cultura hacker en sus contextos. Las investigaciones y acciones citadas en el apartado anterior nos dan pistas valiosas sobre estos principios y nos atrevemos, aunque en una versión beta, señalarlos en este articulo:

1. Hacer por pasión: los hackers no hacen algo simplemente 'por hacer' sino porque eso es interesante para él, o sea, en alguna medida aquello despierta en él una pasión (HIMANEN, 2001). Los profesores y los alumnos deben también conducirse por actividades apasionantes para ellos. Construir un ambiente que favorezca la diversión es algo importante (AGUADO et al., 2015; ESCAÑO, 2018a).

2. Tener libertad como algo fundamental: la libertad esta directamente ligada a la ética hacker en relación al trabajo (HIMANEN, 2001). La escuela debe tener una "una arquitectura que favorezca la libertad y el trabajo colectivo" (PRETTO, 2015, p. 19). 
3. Respeto y estímulo a la diversidad: la diversidad de saberes, culturas y conocimientos es una gran riqueza de las comunidades hacker así como de las escuelas y otros espacios educativos. Esta diversidad debe ser comprendida y respetada (PRETTO, 2015).

4. Cuidado con los otros: es un valor esencial para sustentación de una comunidad (HIMANEN, 2001). Directamente ligado al cuidado está la percepción de que eres parte de una sociedad y una comunidad mayor que sí mismo y eso implica en responsabilidades ante todo este cuerpo social (AGUADO et al., 2015; ESCAÑO, 2018a).

5. Acceso total a los medios: el surgimiento de las primeras comunidades hacker se dio porque estos tuvieron acceso a las computadoras que surgían (LEVY, 1994). El acceso a medios de aprendizaje como computadoras, libros e Internet es un elemento esencial para una educación hacker (ESCAÑO, 2018b; PRETTO, 2015).

6. Acciones, informaciones y decisiones abiertas y compartidas: acciones, informaciones y decisiones impregnadas por una postura abierta hacen todo el proceso más democrático y participativo sirviendo incluso de combustible para que todos se sientan actores en la construcción de ese proyecto. El compartir información es positivo y poderoso, no sólo una postura aconsejable, sino un impositivo para los hackers (AGUADO et al., 2015; BURTET, 2014; ESCAÑO, 2018a; PRETTO, 2015).

7. Acción frente a la pasividad: de hecho, la educación hacker es una educación que estimula la acción. Para los hackers la pasividad debe ser despreciada (HIMANEN, 2001). Se espera del hacker una postura activa ante la red, al grupo y su comunidad. Esto no da espacio para una educación bancaria y cómoda. Los saberes se comparten en la práctica, en la acción y participación creativa (BURTET, 2014; ESCAÑO, 2018a).

8. Despenalización y incentivo del error: el error tiene una importancia singular en el proceso de aprendizaje de los hackers. La criminalización del error debe ser rechazada y más que eso, la oportunidad de errar debe ser estimulada (PRETTO, 2015).

9. Estímulo a la creatividad: la creatividad aquí citada no se asemeja a "tener ideas buenas", sino más que eso, se refiere al uso imaginativo de las habilidades que cada uno tiene para que, superando a sí mismo, pueda crear de forma a contribuir genuinamente para el mundo, su comunidad y red (ESCAÑO, 2018a, 2018b; HIMANEN, 2001).

10. Favorecer la curiosidad: la curiosidad es a menudo lo que motiva al hacker a abrir las cosas, querer comprender cómo funcionan los mecanismos y las estructuras. Favorecer y fomentar este proceso es esencial en la educación (BURTET, 2014). 
11. Ser una educación activista: es parte de la educación hacker comprender que la educación es un proceso de construcción contextualizada, donde la preocupación por los problemas que afectan a sí mismos y otros extrapolan la indignación pasiva y se materializan en una postura activista que ocupa de forma inteligente los espacios digitales y materiales (ESCAÑO, 2018a; MENEZES, 2018).

12. Estímulo a la copia, reutilización y remix: la construcción a partir de lo que ya existe es una práctica común en el día a día, pero fuertemente penalizada en la educación formal. En los colectivos hacker la creación a partir del remix de cosas que ya existen es algo muy característico (ESCAÑO, 2018a; PRETTO, 2015).

13. Postura crítica: este es uno de los aspectos centrales de la educación hacker y esta directamente vinculada a la búsqueda de diversidad en la construcción de opiniones y saberes. Es importante leer el mundo con cierta criticidad aún más en un contexto con tantas fake news. Esta construcción del pensamiento crítico, vinculada a la comprensión que de todos, alumnos, profesores y comunidad, son hacedores de cultura, es la llama principal de la educación hacker como estrategia de empoderamiento y emancipación (ESCAÑO, 2018a, 2018b), donde las jerarquías y estructuras de poder deben ser cuestionadas (PRETTO, 2015).

14. Extrapolar los espacios determinados: ser hacker se refiere también a un modo de vivir en el mundo y una comprensión de que los espacios no son fragmentados (ESCAÑO, 2018a). La escuela debe ser parte de una comunidad y así debe ser vista. Lo mismo pasa con los alumnos y profesores que deben ser entendidos como seres complejos (con sentimientos, emociones, familias, problemas, deseos, etc). La educación hacker extrapola su espacio físico y busca contemplar las diversas dimensiones de lo humano.

15. Ser una educación política: la educación hacker es también política, comprendiendo política como algo no partidista, sino el proceso por el cual las personas construyen consenso a través del diálogo plural y de la preocupación con el colectivo. Esta es una de las caras de la educación hacker segundo Menezes (2018) y un aspecto muy presente en las comunidades de Europa y América Latina (DAVIES, 2017).

Estos principios están de alguna manera interrelacionados y cruzan los diferentes ámbitos de la acción educativa, presentándose como un motor de autorreflexión para los docentes, estudiantes, directores y actores públicos, así como pueden ser ejes estructurales en el desarrollo de metodologías, planes de estudio, políticas educativas y políticas públicas para la educación. Es importante enfatizar que este conjunto de principios no son estáticas, tampoco determinantes. Las investigaciones en relación a la educación hacker sólo están en su inicio y los diferentes proyectos 
que surgen al rededor del mundo aún enriquecerán mucho nuestros debates y construcciones colectivas.

El objetivo principal de este artículo fue proponer una reflexión sobre la educación hacker, presentándola como una expresión emergente de la pedagogía crítica para la sociedad en red. Así como las investigaciones y acciones presentadas en este artículo sirven de inspiración en la búsqueda de una educación cada vez más hacker, los quince principios sirven de orientación para un camino reflexivo en las diferentes dimensiones de la acción educativa y para los diferentes actores.

Nuevas ediciones del proyecto Joven Hacker están planificadas, así como el proyecto Conexão Escola Mundo está en plena ejecución. En muchos lugares del mundo han surgido otras acciones y quizás vendrán muchas más. A través de estas investigaciones y acciones esperamos ampliar nuestra percepción empírica sobre la educación hacker tanto en la educación formal como en la educación no formal, así como tener respuestas más efectivas para las inquietudes contemporáneas expuestas al inicio de este articulo. 


\section{REFERENCIAS}

AGUADO, A. G. O movimento do software livre e suas contribuições para a formação de redes de colaboração na educação. Limeira, SP: Universidade Estadual de Campinas, Faculdade de Tecnologia, 2012.

AGUADO, A. G. et al. Educação Hacker: Uma proposta para formação docente. Revista Inovaeduc, v. 3, 2015.

AMIEL, T. et al. Dominando para não ser dominado: Autonomia tecnológica com o Projeto Jovem Hacker. WORKSHOP INTERNACIONAL DE SOFTWARE LIVRE. Anais... Porto Alegre: 2015 Disponible en: <http://wsl.softwarelivre.org/2015/ 0005/>. Acesso em: 13 set. 2019.

BERNERS-LEE, T. I invented the web. Here are three things we need to change to save it | Tim Berners-Lee. Disponível em: <http://www.theguardian.com/technology/2017/mar/11/tim-berners-lee-web-inventor-save-internet>. Acceso en: 13 set. 2019.

BURTET, C. G. Os saberes desenvolvidos nas práticas de um hackerspace de Porto Alegre. Porto Alegre: Escola de Administração, Universidade Federal do Rio Grande do Sul, 2014.

CADWALLADR, C. 'I made Steve Bannon's psychological warfare tool': meet the data war whistleblower. Disponível em: <https://www.theguardian.com/news/2018/mar/17/data-war-whistleblower-christopher-wylie-faceook-nix-bannon-trump>. Acceso en: 7 jun. 2019.

CASTELLS, M. A Galáxia Internet: reflexões sobre a Internet, negócios e a sociedade. Rio de Janeiro: Jorge Zahar, 2003.

DAVIES, S. R. Hackerspaces: making the maker movement. Cambridge, UK: Polity Press, 2017.

ESCAÑO, C. Cultura libre digital, procomún y educación. Prácticas educativas de cooperación cultural en red por el desarrollo social del procomún digital. II Congreso Internacional Move. net sobre Movimientos Sociales y TIC (2018), p 80-91. Anais...Grupo Interdisciplinario de Estudios en Comunicación, Política y Cambio, 2018a Disponible en: <https://idus.us.es/xmlui/handle/ 11441/70621>

ESCAÑO, C. Educación hacker: una pedagogía crítica (inter) creativa para los comunes del conocimiento. In: La otra educación. Pedagogías críticas para el siglo XXI. Madrid: Universidad Nacional de Educación a Distancia, UNED, 2018b. p. 53-64.

EVANGELISTA, R. A. Para além das máquinas de adorável graça: Cultura hacker, cibernética e democracia. São Paulo: Edições Sesc, 2018.

FREIRE, P. Educação como prática da liberdade. Rio de Janeiro: Paz e Terra, 1967.

FREIRE, P. Pedagogia da indignação: cartas pedagógicas e outros escritos. Rio de Janeiro: Paz e Terra, 2000. v. 5

HIMANEN, P. A ética dos hackers e o espírito da era da informação: a diferença entre o bom e o mau hacker. Rio de Janeiro: Campus, 2001.

INTERNET WORLD STATS. World Internet Users Statistics and 2019 World Population Stats. Disponível em: <https://www.internetworldstats.com/stats.htm>. Acceso en: 7 maio. 2019.

LAPA, A.; PRETTO, N. D. L. La comunicación en disputa: el rol de educadores y científicos. REDU. Revista de Docencia Universitaria, v. 17, n. 1, p. 33-43, 2019.

LEVY, S. Hackers: Heroes of the Computer Revolution. New York, NY, USA: Dell Pub., 1994.

MAXIGAS, P. Hacklabs and hackerspaces: Tracing two genealogies. Journal of Peer Production, n. 2, 2012.

MCLAREN, P. La vida en las escuelas: una introducción a la pedagogía crítica en los fundamentos de la educación. 1. ed. Ciudad del México: Siglo XXI, 2005.

MCLAREN, P.; ESCAÑO, C.; JANDRIĆ, P. Por una pedagogía crítica digital. Retos y alfabetización en el S. XXI. In: La otra educación. Pedagogías críticas para el siglo XXI. Madrid: Universidad Nacional de Educación a Distancia, UNED, 2018. p. 3554.

MENEZES, K. M. Pirâmide da pedagogia hacker=[vivências do (in) possível]. Salvador, BA: Universidade Federal da Bahia, 2018.

PRETTO, N. D. L. Hackear a educação. Revista Facta, p. 74-81, abr. 2015.

RAYMOND, E. The cathedral and the bazaar. Knowledge, Technology \& Policy, v. 12, n. 3, p. 23-49, 1999.

RAYMOND, E. Jargon File Resources. Disponível em: 〈http://www.catb.org/jargon/>. Acceso en: 1 jun. 2018.

SCHROCK, A. R. "Education in Disguise": Culture of a hacker and maker space. InterActions: UCLA Journal of Education and Information Studies, v. 10, n. 1, 2014.

SINGER, N. How Google Took Over the Classroom. The New York Times, 13 maio 2017.

SOURCEFORGE COMMUNITY. SourceForge - Download, Develop and Publish Free Open Source Software. Disponible en: <https://sourceforge.net/>. Acesso em: 11 jun. 2019. ZUBOFF, S. Big other: surveillance capitalism and the prospects of an information civilization. Journal of Information Technology, v. 30, n. 1, p. 75-89, 2015. 


\section{RESUMEN}

Este artículo propone una reflexión sobre la educación hacker, presentándola como una expresión emergente de la pedagogía crítica para la sociedad en red. Partiendo de la importancia de poner en discusión en los espacios educativos las dinámicas de poder que saltan de las redes e influyen en la vida de las personas, vuelve su mirada hacia el movimiento hacker y su forma de hacer educación. A partir de la revisión de investigaciones, acciones y proyectos relacionados con el tema en los últimos seis años, se proponen quince principios de la educación hacker, los cuales se ponen tanto como conductores para la autorreflexión de docentes, estudiantes, directores y actores públicos, como ejes estructurales en el desarrollo de metodologías, planes de estudio y políticas educacionales.

Palabras clave: Educación hacker; pedagogía crítica; sociedad en red

\section{EDUCAÇÃO HACKER: UMA EXPRESSÃO EMERGENTE DA PEDAGOGIA CRÍTICA PARA A SOCIEDADE EM REDE}

\section{RESUMO}

Este artigo propõe uma reflexão acerca da educação hacker, apresentando-a como uma expressão emergente da pedagogia crítica para a sociedade em rede. Partindo da importância de se colocar em discussão nos espaços educativos as dinâmicas de poder que saltam das redes e influenciam a vida das pessoas, volta-se o olhar para o movimento hacker e seu modo de fazer educação. A partir da revisão de pesquisas, ações e projetos relacionados ao tema nos últimos seis anos, são propostos quinze princípios da educação hacker, os quais podem se colocar tanto como aspectos condutores para a autorreflexão de professores, alunos, diretores e atores públicos, como possíveis eixos estruturantes no desenvolvimento de metodologias, currículos e políticas para a educação.

Palavras-chave: Educação hacker; pedagogia crítica; sociedade em rede

\section{HACKER EDUCATION: AN EMERGING EXPRESSION OF CRITICAL PEDAGOGY FOR THE NETWORK SOCIETY}

\section{Abstract}

This article proposes a reflection about the hacker education, presenting it as an emerging expression of critical pedagogy to the network society. Starting from the importance of bring for discussion in the educational spaces the power relations that leap from the networks and influence people's lives, turns his gaze to the hacker movement and its way of doing education. From the review of researches, actions and projects related to the theme in the last six years, fifteen principles of hacker education are proposed, which can be considered as conductive aspects for the self-reflection of teachers, students, principals and public actors, as well as possible structuring axes in the development of methodologies, curricula and educational policies.

Keywords: Hacker education; critical pedagogy; network society

Submetido em Março de 2019 Aprovado em Outubro de 2019 